\title{
The Effect of Upstream Duct Boundary Layer Growth and Compressor Blade Lean Angle variation, on an Axial Compressor Performance.
}

\author{
I Templalexis $^{1, *}$, V Pachidis $^{2}, \mathbf{P}$ Pilidis $^{2}$, and $\mathbf{P}$ Kotsiopoulos ${ }^{1}$ \\ ${ }^{1}$ Hellenic Air Force Academy, Department of Aeronautical Sciences, Section of Thermodynamics, Power and \\ Propulsion Systems, Hellenic Air Force, Dekelia Air Base, Greece \\ ${ }^{2}$ Cranfield University, School of Engineering, Department of Power and Propulsion, Gas Turbine Engineering \\ Group, Cranfield, Bedfordshire, MK43 0AL, England
}

\begin{abstract}
The gas turbine engine's compressor is extremely vulnerable on upstream duct induced flow nonuniformities, whether this is the engine intake or an interconnecting duct. That is justified by its position being literally an extension of the duct flow path, coupled to the fact that it operates under adverse pressure gradients. In particular this study focuses on performance deviations, between an installed and an uninstalled compressor. Test results acquired from a test bed installation will differ from these recorded when the compressor operates as an integral part of an engine. The upstream duct, whether an intake or an inter-stage duct, will affect the flow field pattern ingested into the compressor. The case study presented here aims mostly to qualify the effect of boundary layer growth along the upstream duct walls, upon compressor performance. Additionally, compressor performance response on blade lean angle variation is being addressed, with the aim of acquiring an understanding as to how compressor blade lean angle changes interact with intake induced flow non uniformities. Such studies are usually conducted as part of the preliminary design phase. Consequently, experimental performance investigation is excluded at this stage of development and therefore computer aided simulation techniques are between the few if not the only option for compressor performance prediction. Given the fact that many such design parameters need to be assessed under the time pressure exerted by the tight compressor development program, the compressor flow simulation technique needs to provide reliable results while consuming the least possible computational time. Such a low computational time compressor flow simulation method, among others, is the two dimensional (2D) streamline curvature (SLC) method, being also applied within the frame of reference of the current study. The paper is introduced by a brief discussion on SLC method. Then a reference is made to the Radial Equilibrium Equation (REE) which is the mathematical basis of SOCRATES the turbomachinery flow simulation tool that was used for this study. Subsequently the influence of the upstream duct on the compressor inlet radial flow distribution is being addressed, with the aim of adjusting compressor blade inlet lean angle, in order to minimize compressor performance deterioration. The paper is concluded with a discussion of the results.
\end{abstract}

Keywords: Lean Angle, Intake, Streamline Curvature, Radial Equilibrium Equation, Compressor Performance.

* Corresponding author: Lecturer, Department of Aeronautical Sciences, Section of Thermodynamics, Power and Propulsion Systems, email: i.templalexis@yahoo.gr 


\section{INTRODUCTION}

Given the fact that in most of the cases, gas turbine engine's compressors handle subsonic flow, their performance is affected by the upstream engine intake or compressor interconnecting duct. Such component interaction effects, should be assessed and thus be taken into account, during the preliminary design phase of the compressor. Alternatively, design modifications may need to be introduced to an existing compressor design that is installed behind a new duct design. The aim in this former case is to prevent compressor performance degradation due to installation effects.

Compressor upstream components affect the compressor performance in two distinct ways: i) Flow-field thermodynamic and momentum distortion, ii) Boundary layer ingestion. There has been a vast amount of research effort being invested in the past, in order to predict the impact of the flow field distortion on compressor performance. References $[\mathbf{1 , 2 , 3}]$ are just a few citations in the field. On the other hand compressor key design variables like the blade lean angle [4,5], have also been assessed on the basis of their impact on compressor performance.

The current paper addresses the effect of the blade lean angle on an axial compressor's performance as well as the compressor's response to the blockage introduced by the ingested boundary layer. These effects are firstly examined separately against a reference datum. Subsequently their superimposed impact is compared against their cumulative impact on compressor performance.

The work was carried out using SOCRATES a 2D SLC based, compressor performance prediction and design tool. Given the character of the tool, a fast in-viscid flow solver incorporating phenomenological models, only the qualitative value of the results can be appreciated. Nonetheless, it remains a valuable tool indicating compressor performance trends during the preliminary design phase, providing thus the designer with important information for compressor candidate design refinement.

\section{STREAMLINE CURVATURE METHOD.}

SLC is a well established through-flow calculation method, having its origins back to the early fifties [6]. It combines the low computational time for convergence due to the nature of the flow being considered (compressible, in-viscid, 2-D flow), with results of adequate engineering accuracy, depending on the set of phenomenological models being used. Flow solvers based on this method, and similar through-flow calculation methods gain nowadays increasing attention as they tend to substitute traditional 0-D tools for compressor preliminary design.

A through-flow solver belongs to the category of SLC solvers when it solves the REE stemming from the application of Newton's second law of motion along a particular direction on a differential flow volume of an in-viscid flow. SLC solvers ended up to consist a category of such methods on their own, because there were several deviations concerning the solution grid definitions, the parallel use of other fundamental laws, the form of the REE and even the mathematical solution scheme. A more profound insight in SLC method and its derivatives, is given in [7].

\section{SIMULATION TOOL.}

The 2D SLC Compressor Software [7,8,9,10], precursor of SOCRATES was used as the flow field simulation tool. It solves the following general form of the REE:

$$
\begin{aligned}
& V_{m} \frac{d V_{m}}{d s}=V_{m}^{2}\left(\begin{array}{l}
\sin -\gamma \tan +\gamma \frac{d \varepsilon}{d m}- \\
-\frac{d \varepsilon}{d s} \frac{\sin -\gamma}{\cos +\gamma} \\
-\frac{\sin \varepsilon \sin -\gamma}{r} \\
-\frac{1}{\rho} \frac{d \rho}{d m} \sin -\gamma+ \\
+\frac{\cos -\gamma}{r_{c}}
\end{array}\right)+ \\
&+V_{m}\left(\begin{array}{l}
\tan \lambda \frac{d W_{w}-1}{d m}+ \\
+2 \omega \sin \varepsilon \tan \lambda
\end{array}\right)+ \\
&+\left(\begin{array}{l}
W_{w} \frac{d W_{w}-2 \omega W_{w} \cos \gamma+}{d s}-2 \\
+\frac{d I}{d s}-T \frac{d S}{d s}+ \\
T \frac{d S}{d m}\left(\begin{array}{l}
\sin -\gamma \cos { }^{2} \beta- \\
-\tan \lambda \sin \beta \cos \beta
\end{array}\right)
\end{array}\right)
\end{aligned}
$$

which is basically a second order linear differential equation with respect to the meridional velocity of the following form:

$$
V_{m} \frac{d V_{m}}{d s}=A V_{m}^{2}+B V_{m}+C
$$

where $\mathrm{A}, \mathrm{B}$ and $\mathrm{C}$ constants are as follows:

$$
\begin{aligned}
& A=\left(\begin{array}{l}
\sin -\gamma \tan +\gamma \frac{d \varepsilon}{d m}- \\
-\frac{d \varepsilon}{d s} \frac{\sin -\gamma}{\cos +\gamma}-\frac{\sin \varepsilon \sin -\gamma}{r} \\
-\frac{1}{\rho} \frac{d \rho}{d m} \sin -\gamma+\frac{\cos -\gamma}{r_{c}}
\end{array}\right) \\
& B=\left(\tan \lambda \frac{d W_{w}}{d m} \frac{1}{r}+2 \omega \sin \varepsilon \tan \lambda\right)
\end{aligned}
$$




$$
C=\left(\begin{array}{l}
-\frac{W_{w}}{r} \frac{d}{d s}-2 \omega W_{w} \cos \gamma+ \\
+\frac{d I}{d s}-T \frac{d S}{d s}+ \\
T \frac{d S}{d m}\left(\begin{array}{l}
\sin -\gamma \cos ^{2} \beta- \\
-\tan \lambda \sin \beta \cos \beta
\end{array}\right)
\end{array}\right)
$$

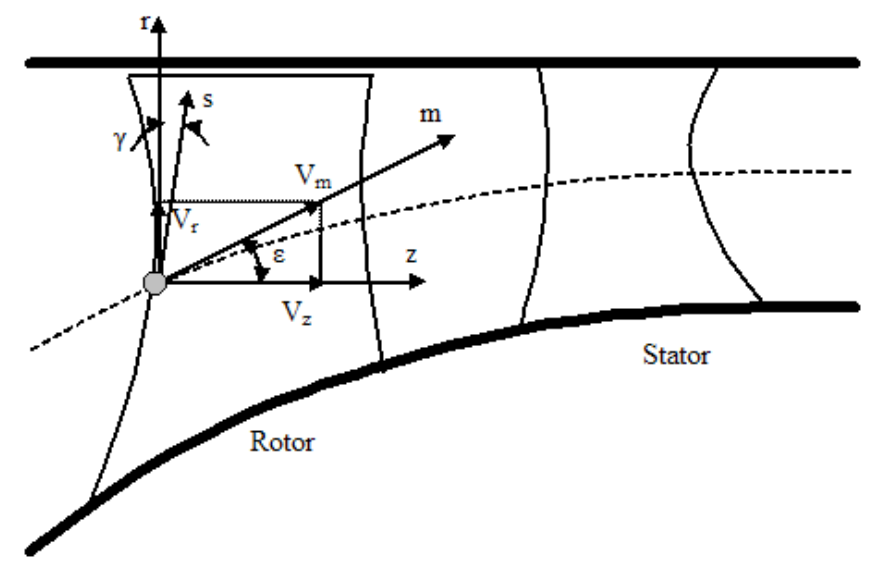

Fig. 1: Meridional plane vector - angle definition.

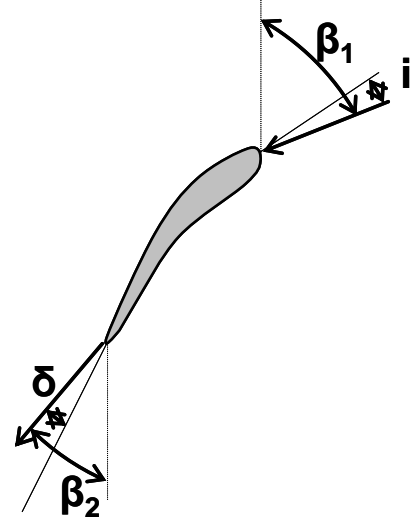

Fig 2: Blade angle definition.

Concerning the current version of the code the following features have been incorporated:

- Dynamic adaptation of the computational grid, taking place in-between iterations, to support fast and stable convergence.

- Extension of the solution scheme to cover duct flow calculations upstream or downstream of the compressor. More details are given in section 4.2.

- Inclusion of a sophisticated dynamic convergence control subroutine.

- Development of a graphical user interface in order to enhance the friendliness of the code against the user.

Angle and vector definitions are demonstrated on figures 1 and 2.

\section{CASE STUDY}

\subsection{Geometry Definition}

The current case study is based on the compressor geometry given in NASA TP 1493 report [11]. It concerns a two stage axial fan (Figure 3, Table 1).

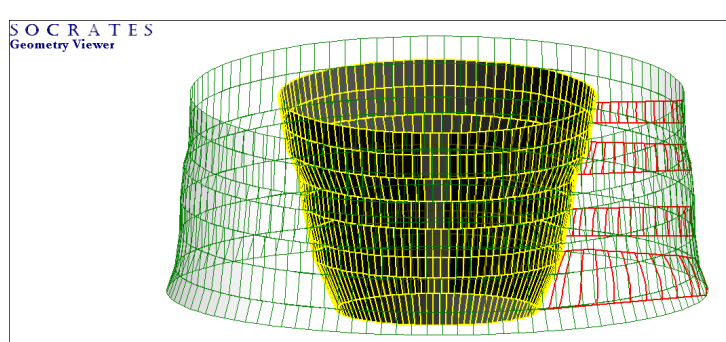

Fig. 3: Two stage fan geometry.

The fan is fitted with a generic divergent intake duct, with an area ratio of 0,7 and a total length of $26 \mathrm{~cm}$ (Figure 4).

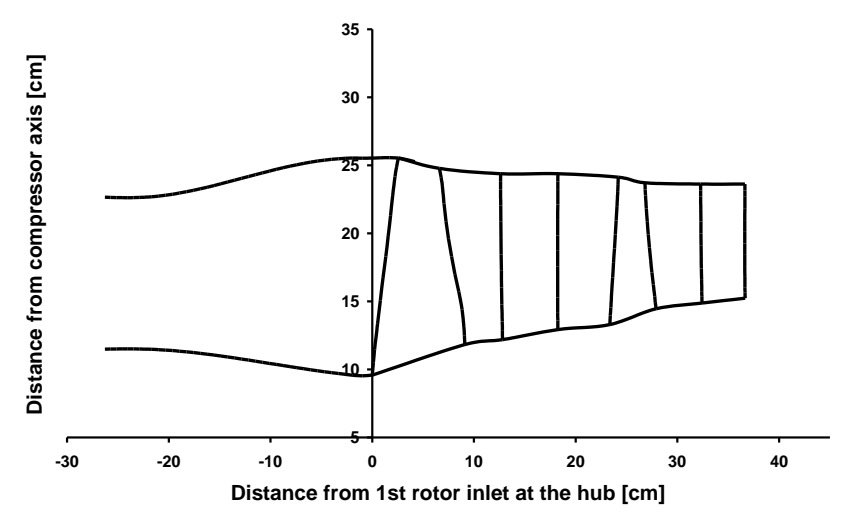

Fig. 4: Intake Compressor geometry.

\begin{tabular}{|l|r|}
\hline Pressure Ratio & 2.399 \\
\hline Isentropic Efficiency & 0.849 \\
\hline Mass Flow [kg/sec] & 33.248 \\
\hline RPM & 16042.80 \\
\hline Inlet Hub-Tip Ratio & 0.375 \\
\hline
\end{tabular}

Table 1: Two-stage fan design overall parameters [11]

\subsection{Solution Scheme}

The REE is solved over the boundaries of each blade row (blade leading and trailing edge), as well as along quasiorthogonal paths within the duct, in order to determine a meridional radial velocity distribution.

During the first iteration and before the flow-field calculation starts, there exists an assumed velocity distribution throughout the compressor as well as a distribution of thermodynamic properties. Consequently, subroutines containing phenomenological models can run individually on a 
separate module to produce a set of empirical factors which are then fed to the next iteration. In figure 5 the initial computational grid is demonstrated.

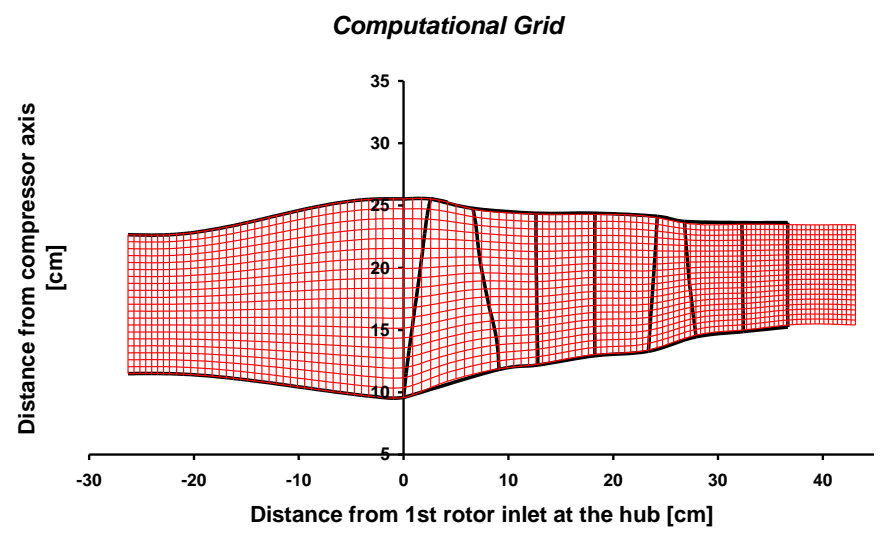

Fig. 5: Intake - Compressor geometry grid.

A set of boundary conditions (total temperature, total pressure, absolute Mach number and absolute flow angle) are specified at the intake throat. There are no specific boundary conditions specified at the compressor outlet plane. The position of streamlines is fixed across the inlet plane. Varying the inlet Mach number distribution, a range of mass flow values along a constant speed line may be obtained. Finally an extra boundary condition is specified: the blockage factor value that accounts for the boundary layer ingested into the intake.

\subsection{Case Study Phenomenological Models}

As mentioned in section 3, all necessary input data for the empirical models are readily available in between iterations. The development of the code from now on is split in two parallel modules. The first one deals with the in-viscid flow calculation and the second, consisting a stand-alone separate module, provides the in-viscid solver with the empirical factors in order for the viscous nature of the flow to be introduced. In solution it should be mentioned that each empirical models package, should be complete in terms of viscous effects description, as the in-viscid part of the code will only use one such package during execution. The code had to be structured in such a way, because there is not a universally acceptable book keeping system of the losses. In other words, phenomenological models modules, cannot exchange specific models (the profile loss model for instance between two different modules) in order to avoid confusions.

Concerning the current case study the phenomenological model module contains the following set of empirical models:

- For the blade incidence minimum loss calculation, Lieblein's (1965) approach is adopted. [12].

- For the blade minimum loss deviation calculation, Carter's rule was used [13].

- The calculation of the off-design deviation angle was done according to Creveling [14].
- For the determination of losses the approach given by Miller [15] was adopted. According to Miller et al., it is assumed that the total pressure loss of the blade row is the result of the interaction of different loss components, i.e. profile losses, secondary losses and shock losses, which are considered to act through independent mechanisms. Each mechanism is reflected by a corresponding loss factor and the total loss factor is given by their sum. Blade profile loss is determined according to Swan's model, [16] the shock losses are calculated as described by Schwenk [17] and secondary losses are determined according to Griepentrog's approach. [18].

- The end wall boundary layer calculations for both the bladed and bladeless space are being performed according to the model described in reference [19]

\subsection{Case Study Set Up}

Table 2 summarizes the boundary conditions of the case study under context. Radial distribution for all the parameters is assumed constant since the impact of radially distorted conditions at the intake throat plane does not interest the current study. Mach number range is determined on the basis of establishing a safe mass flow window well contained between chocking and surge boundaries. Compressor speed was held constant in all cases and equal to approximately $8000 \mathrm{rpm}$. A part load condition has been selected given the fact that this is a very demanding case scenario from the simulation point of view. Blockage factor range was taken quite wide in order to cover any extreme case of boundary layer separation due to shock presence, steep climb conditions, etc. Additionally the study aimed to address to qualitative response of the compressor and it did not necessarily referred to an actual case. Finally the compressor blade lean angle was varied between 5 degrees of backward (suction blade surface facing the upper casing wall) to 5 degrees of forward (pressure blade surface facing the upper casing wall) leaned blades, for both compressor stages (see figure 6 for lean angle definition). In all cases the theoretical reference datum is considered to be the case of 0 lean angle and a blockage factor of 1 (no blockage).

\begin{tabular}{|l|r|}
\hline Total Temperature & $288 \mathrm{~K}$ \\
\hline Total Pressure & $1,013 \mathrm{bar}$ \\
\hline Mach Number & $0,5-0,7$ \\
\hline Air Absolute Angle & $0 \mathrm{deg}$ \\
\hline Blockage Factor & $1-0,96$ \\
\hline Inlet Hub-Tip Ratio & 0.375 \\
\hline
\end{tabular}

Table 2: Intake Throat Boundary Conditions 


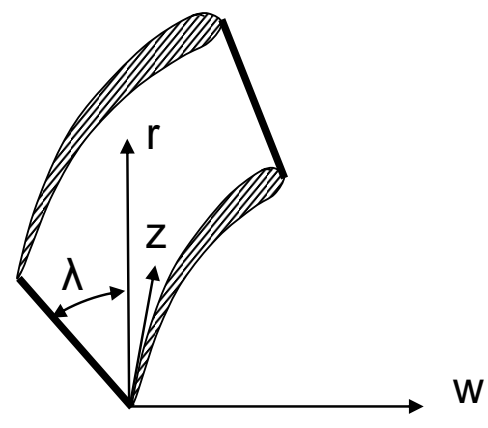

Fig. 6: Lean angle definition.

\subsection{Case Study's Solver Validation.}

The solver has been validated against radial meridional velocity profiles, for both viscid and in-viscid operating modes. The experimental reference results were taken from NASA TP 1493 report [11] for the setting of around $8000 \mathrm{rpm}$. In order to validate the in-viscid operating mode the loss factors appearing in the experimental set of data were used excluding thus from the simulation the phenomenological models' effects (Figures $7,8)$.

\section{Meridional Velocity Profile Blade Leading Edge}

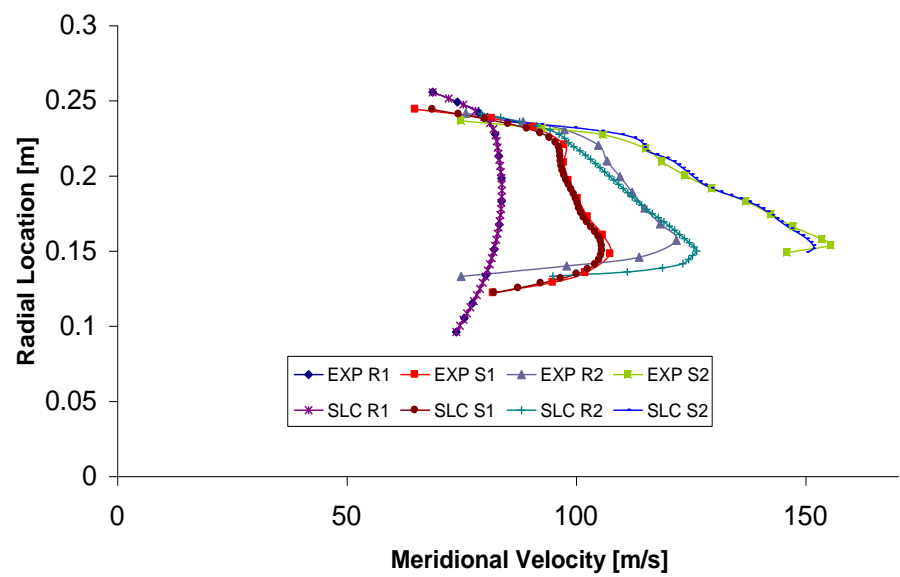

Fig. 7: Leading edge meridional velocity profiles comparison between experimental data and in-viscid solver
Meridional Velocity Profile Blade Trailing Edge

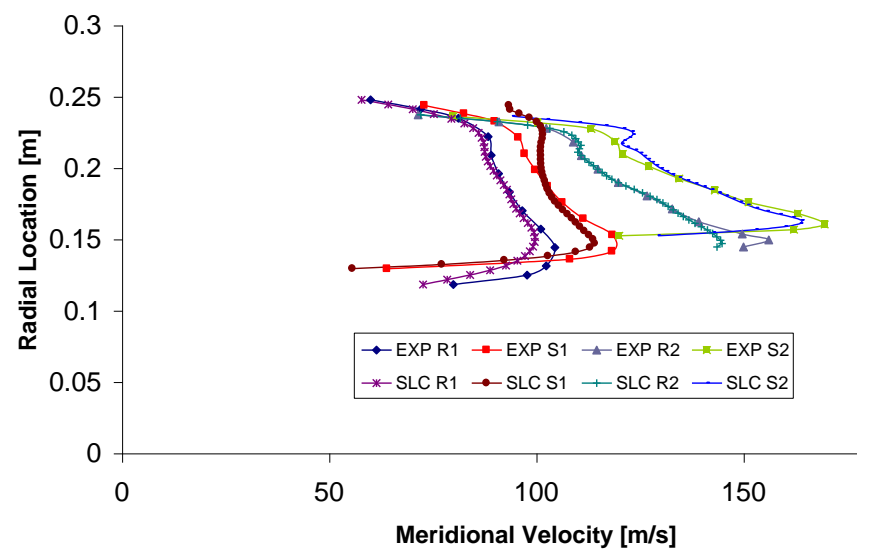

Fig. 8: Trailing edge meridional velocity profiles comparison between experimental data and in-viscid solver

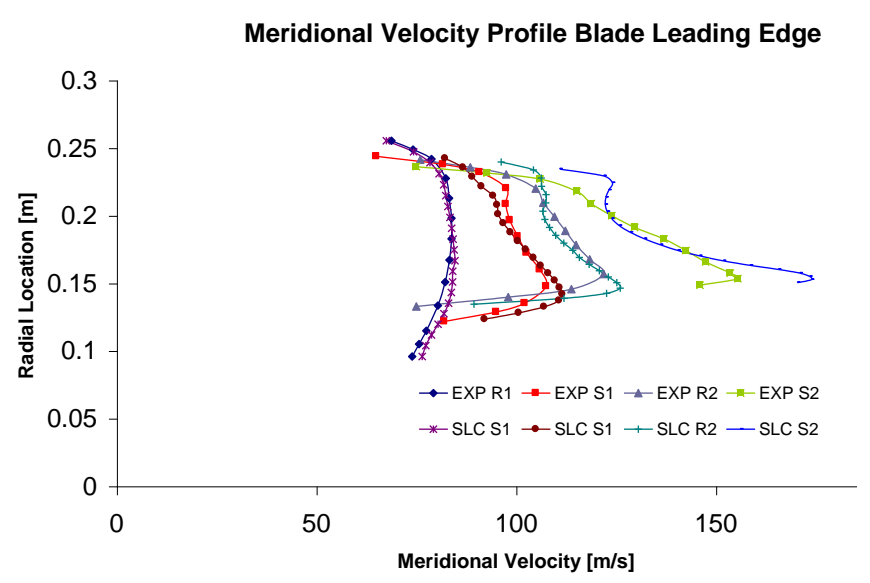

Fig. 9: Leading edge meridional velocity profiles comparison between experimental data and phenomenological module. 


\section{Meridional Velocity Profile Blade Trailing Edge}

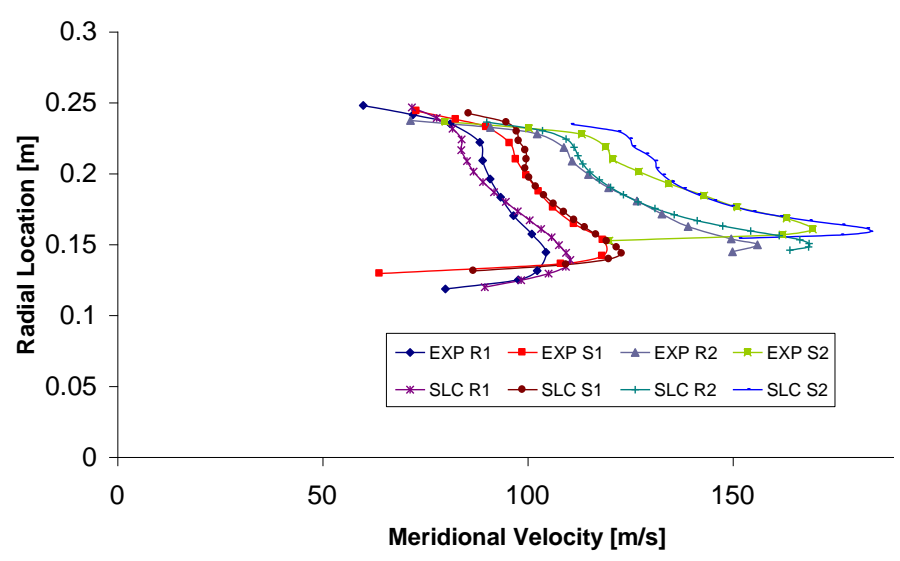

Fig. 10: The trailing edge meridional velocity profiles comparison between experimental data and phenomenological module

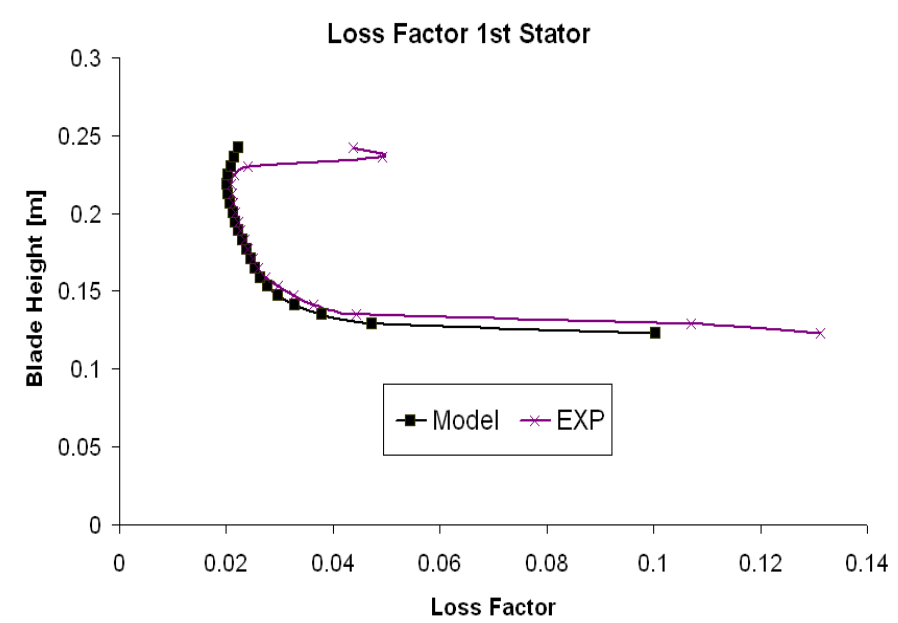

Fig. 11: Loss factor comparison - 1st stage stator

As a second step, the loss factors were calculated according to the models presented earlier and the corresponding results regarding the meridional velocity profiles are presented in figures 9 and 10. The 1st's row stator loss factors (figure 11) are shown, as an indicative case as to how calculated and experimental values compare to each other. Comparison shows an adequate agreement especially in the mid-span blade region. The great deviations spotted at the far extreme points at the blade root and tip regions, are due to the fact that measured values come from within the boundary layer where profile loss models usually perform the worse.

As far as the in-viscid mode is concerned, it can be seen that agreement between calculated and experimental meridional velocity profiles is very satisfactory. The quantitative trends are captured at all positions, including the sharp turns on the velocity curves which usually cause convergence instabilities.
Concerning the performance of the set of phenomenological models, despite the fact that the compressor is simulated away from the design point, results were also satisfactory. The biggest deviations between the two profiles were recognized to be at the leading and trailing edge of the last stator. This does not necessarily means that the models perform the worst over this last compressor blade row. Certain percentage of this mismatch comes as a cumulative error of the upstream rows.

Convergence time for both cases did not exceed 30 seconds for a 21 streamline setting and an error tolerance for streamtube mass flow at the level of $10^{-4}$. The total number of iterations (an iteration here is defined as a complete calculation throughout the compressor) in the first case was 8 , where as for the later case where the loss factors were calculated by the phenomenological models module, the code needed 13 iterations before convergence was achieved. The code run in a core duo Pentium, 1,8 MHz processor.

\section{DISCUSSION OF THE RESULTS}

\subsection{Lean Angle Variation}

Lean angle was varied in the circumferential direction as shown in figure 6. Backward leaning is defined when the suction surface is facing on the end-wall. Lean angle was set to 5 degrees for both cases, a constant value for all blade rows. The reference line for this case was the compressor performance for zero lean angle (unleaned blades). Mass flow was again varied through the intake throat Mach number variation over the same range as it was done for the case of blockage factor variation. A thorough study concerning the effect of blade lean angle on a transonic fan is given in reference [5]. Compressor setting, number of streamlines and stream-tube mass flow tolerances were the same as in the case of blockage factor variation. Total number of iterations and convergence times were also at the same level. The results are demonstrated on figures 12 and 13 .

Qualitative trends of the pressure ratio curves were in accordance to these given in [5] by Denton and $\mathrm{Xu}$, produced for an axial fan by a 3D multistage viscous solver. Forward leaning caused a reduction in pressure ratio, being much more pronounced than the corresponding increase caused in pressure ratio, by the opposite lean angle setting. Deviations of efficiency curves are expected to arise from blade loading alternations, based on flow turning. In this specific case backward lean did not push the compressor to operate into the area of increased profile losses. On the other hand forward leaning demonstrated, over the entire mass flow range, a reduction in efficiency which is magnified in higher pressure ratios where velocities are also higher. 


\section{Lean Angle Variation}

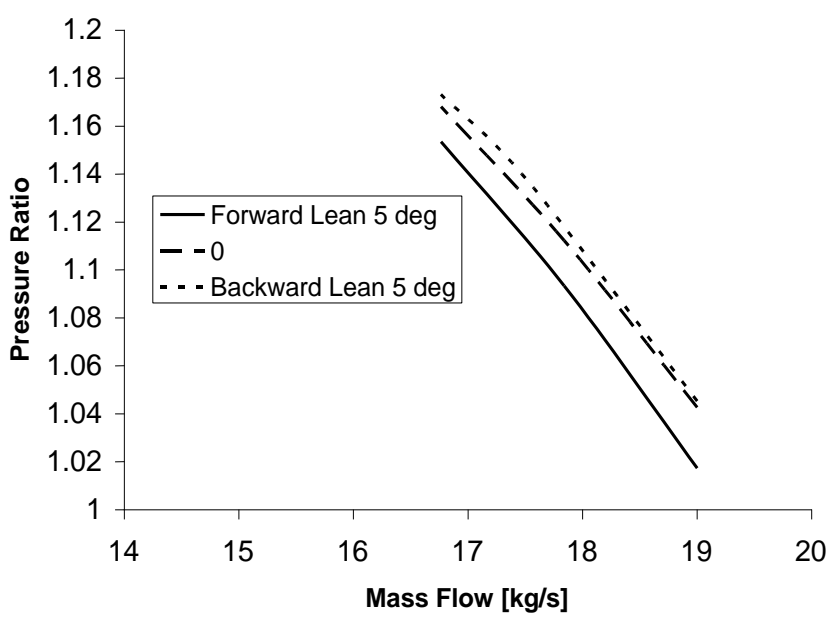

Fig. 12: Compressor characteristic - Pressure ratio for lean angle variation.

Lean Angle Variation

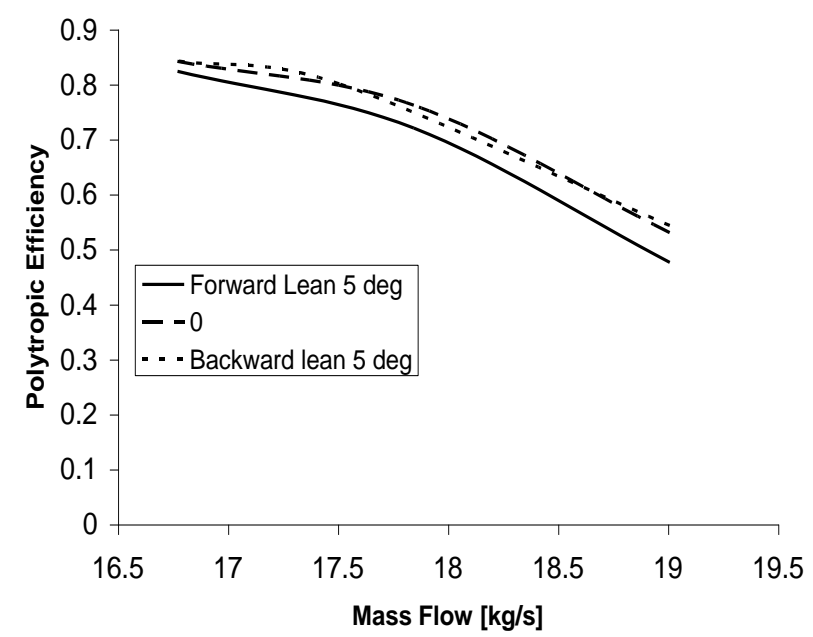

Fig. 13: The Compressor characteristic - Polytropic Efficiency for lean angle variation.

\subsection{Blockage Factor Variation}

Blockage factor at the intake throat was varied from 1 to 0,96 . The Mach number over the same plane was varied from 0,5 to 0,7 to get the range of mass flow appearing on the graphs (Figure 14 and 15). Calculation without flow blockage (blockage factor equals to 1), is used as the reference line. Pressure ratio at a certain mass flow setting, is eventually reduced due to the higher axial velocities caused by the reduced flow area. The simulation was not pushed towards the surge region of the map, as it was not a subject of interest for the current study.

Another qualitative remark worth mentioning is that the range of pressure ratios at the left end of the curves is narrower than the corresponding range at the right end. This can be justified by the great variation of polytropic efficiency at higher mass flows. It should be barred in mind that losses at these compressor settings, where Mach numbers are well below critical value, are principally due to profile losses. A higher velocity variation caused at higher mass flows, is mainly responsible for the deviation of the efficiency curves. In all cases variations in pressure ratio did not exceed $5 \%$ reduction.

Convergence was achieved after 13 to $\max 33$ iterations depending on the case, demonstrating a maximum convergence time of no more than 1 minute in a core duo Pentium, $1,8 \mathrm{MHz}$ processor. Twenty one streamlines were used to cover the compressor annulus and the convergence tolerance for the stream-tube mass flows was set to $10^{-4}$.

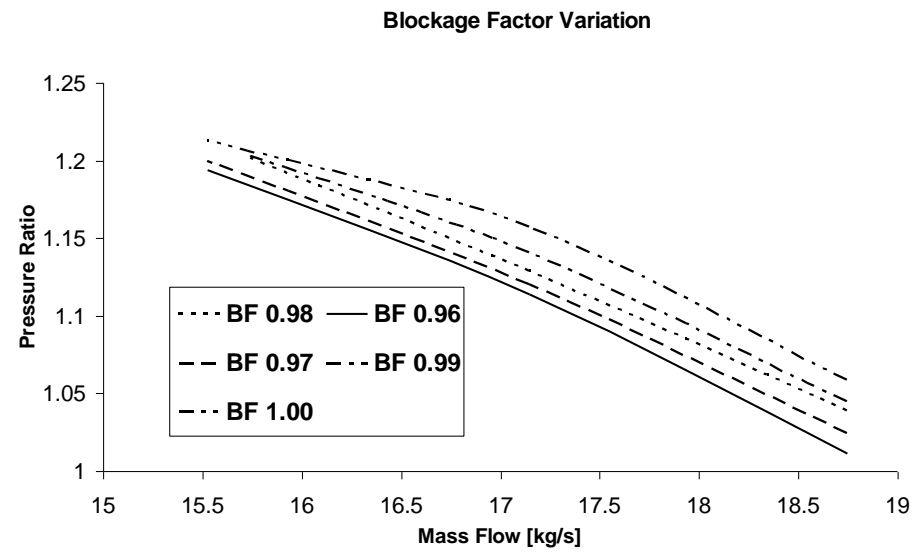

Fig. 14: Compressor characteristic - Pressure ratio for blockage factor variation.

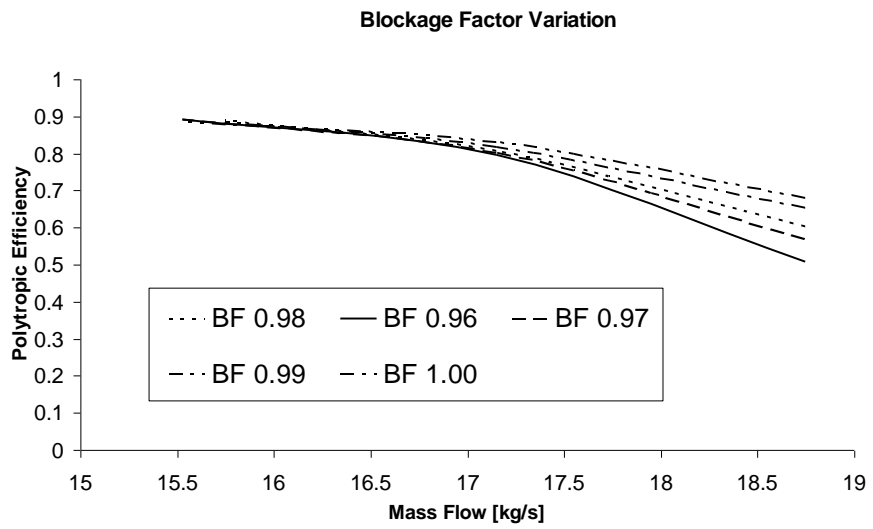

Fig. 15: The Compressor characteristic - Polytropic Efficiency for blockage factor variation.

\subsection{Combined Lean Angle Blockage Effect.}

The last section of this blockage factor - lean angle parametric study, regards the additive and the cumulative effect of the above mentioned parameters. Figure 16 demonstrates the pressure ratio vs. mass flow trends of the two following cases: i) Based on the results of two independently examined cases, 0,98 blockage factor and 5 degrees of forward leaning, the "additive" 
curve was drawn by simply adding algebraically the differences that each case demonstrated from the datum case, (blockage factor equal to 1 and lean angle equal to 0 ) and ii) the "cumulative" curve coming as a direct output of SOCRATES for 0,98 blockage factor and 5 degrees backward leaned blades. The values of blockage factor and lean angle were chosen under the criterion of anticipating the flow blockage effect by the leaning of the blades. The important conclusion that can be drawn from this study, is that for the "cumulative" case the pressure ratio gain by the leaned blades was slightly lower as opposed to what would someone expect by simply adding the differences. However differences were generally marginal meaning that as far as these two effects is concerned, they can be investigated individually and then simply add algebraically the corresponding effects.

\section{Cumulative Lean Angle Blockage Effect}

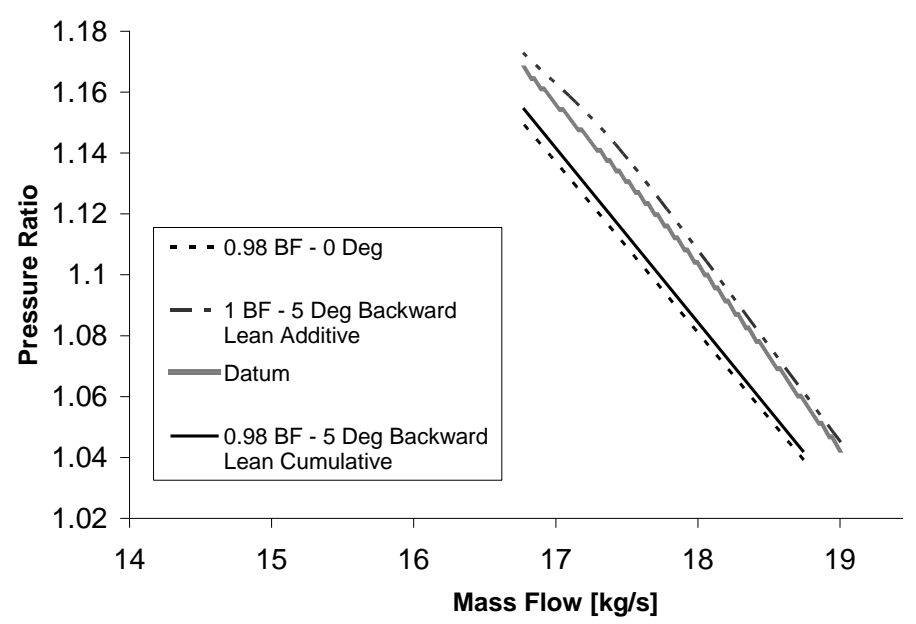

Fig. 16: Cumulative lean angle blockage effect on compressor performance.

\section{CONCLUSIONS}

The current study was based on an experimental two stage fan running at approximately $8000 \mathrm{rpm}$, fitted downstream of an axi-symmetric divergent duct. The study aimed to investigate both the independent and the cumulative effect of an axial compressor blade lean angle variation against the flow blockage generated by an upstream duct. Simulations were conducted using SOCRATES a 2D-SLC compressor software, an in-viscid 2D flow solver incorporating phenomenological models. Both modules, the solver and the models were validated against experimental data on a radial meridional velocity profile basis. Boundary conditions were for all simulation cases applied at the upstream duct inlet section. The Mach number covered a range of 0,5 to 0,7 and the blade span was described by twenty one streamlines in total. The main conclusions drawn out of this study were the following:

- Validation of the "in-viscid" part of the code, demonstrated a very satisfying qualitative and quantitative agreement.
- Validation of the specific phenomenological models module proved that it is adequate for capturing qualitative trends, but actual velocity values, especially close to the blade outer regions (hub and tip) deviated quite substantially.

- Blockage factor caused a reduction in both pressure ratio and efficiency. Those trends were more pronounced at the high mass flow end of the curves.

- Backward leaning proved to be slightly favorable in terms of pressure ratio, whereas forward leaning had the opposite and much more pronounced effect.

- The detrimental effect on compressor pressure ratio due to intake boundary layer ingestion can be counteracted by backward blade lean.

- Blade lean angle variations and upstream boundary layer ingestion, as far as the compressor performance is concerned, can be addressed separately and then add the corresponding effects algebraically.

\section{REFERENCES}

1 Longley, J. P., Greitzer, E., M., 1991, "Inlet Distortion Effects In Aircraft Propulsion System Integration".

2 Calogeras, J., E., Johnsen, R., L., Burstadt, P., L., 1974, "Effect Of Screen Induced Total Pressure Distortion On Axial Flow Compressor Stability." NASA TM X-3017.

3 Milner, E., J., 1977, "Performance And Stability Of A J8513 Compressor With Distorted Inlet Flow.", NASA TM X3515.

4 Choon-Man Jang, Abudus Samad, Kwang-Yong Kim "Optimal Design of Swept, Leaned and Skewed Blades in a Transonic Axial Compressor", GT2006-90384, ASME Turbo Expo, Power For Land, Sea and Air, Barcelona, Spain May 2006.

5 J. D. Denton, L. Xu "The Effects of Lean and Sweep on Transonic Fan Performance", GT2002-30327, ASME Turbo Expo, Power For Land, Sea and Air, Amsterdam, The Netherlands, June 2002.

6 Wu, C. H. , Wolfenstein, L. 1949 "Application Of Radial Equilibrium Condition To Axial-flow Compressor And Turbine Design", NACA TN 1795.

7 Templalexis, I., Pachidis, V., Pilidis, P., and Kotsiopoulos, P., "The Effect of Blade Lean on the Solution of the Radial Equilibrium Equation", GT200850259, ASME Turbo Expo, Power For Land, Sea and Air, Berlin, Germany, June 2008.

8 Templalexis, I., Pilidis, P., Pachidis, V. and Kotsiopoulos, P., January 2008 "Development of a 2D compressor streamline curvature code", Transactions of the ASME, Journal of Turbomachinery, TURBO-06-1178, Vol. 129, Issue 4.

9 Pachidis, V., Pilidis, P., Templalexis, I., Alexander, T. and Kotsiopoulos, P., "Prediction of Engine Performance Under Compressor Inlet Flow Distortion Using Streamline 
Curvature", Transactions of the ASME, Journal of Engineering for Gas Turbines and Power, GTP-05-1192, Vol. 129, p. 97, January 2007.

10 Pachidis, V., Pilidis, P., Templalexis, I., Marinai, L., "An Iterative Method for Blade Profile Loss Model Adaptation Using Streamline Curvature", Transactions of the ASME, Journal of Engineering for Gas Turbines and Power, GTP07-1072, Vol.130, Iss.1, December 2007. ASME Cycle Innovations Committee Best Paper Award for 2007.

11 Urasek D. C., Gorell W. T. and Cunnan W. S., "Performance Of Two-Stage Fan Having Low-AspectRatio, First Stage Rotor Blading", NASA Technical Paper 1493, 1979.

12 Lieblein S., 1965 "Chapter VI - Experimental Flow In Two-Dimensional Cascades", of "NASA SP 36 Aerodynamic Design Of Axial Flow Compressors", Scientific and Technical Information Division, National Aeronautics and Space Administration, Washington DC.

13 Carter A. D. S., 1950 "The Low Speed Performance Of Related Aerofoils In Cascade", National Gas Turbine Establishment, Report No. R.55, September 1949, Reprinted by the Aeronautical Research Council, CP29.

14 Creveling H. F. and Carmody R. H., 1968 "Axial Flow Compressor Computer Program for Calculating Off-Design Performance (Program IV)", General Motors, Allison Division, Indianapolis, Prepared for NASA, Report CR72427.

15 Miller G. R., Lewis, G. W. and Hartmann M. J., 1961 "Shock Losses In Transonic Rotor Rows", Transactions of the ASME, Journal of Engineering for Power, Vol. 83, pages 235-242.

16 Swan, W. C., 1958 "A Practical Method Of Predicting Transonic-Compressor Performance", Journal of Engineering for Power, Transactions of the ASME 83: pp 322-330.

17 Schwenk F. C., Lewis G. W. and Hartman M. J., 1957 "A preliminary analysis of the magnitude of shock losses in transonic compressors", NACA RM E57A30.

18 Griepentrog, H. R. "Secondary flow losses in axial compressors." AGARD LS 39, 1970.

19 Aungier R. H., 2003 "Axial-Flow Compressors: A Strategy For Aerodynamic Design And Analysis", ASME Press, Three Park Avenue, New York, NY 10016, pp 177 - 197.

\section{LIST OF FIGURES}

Fig. 1: Meridional plane vector - angle definition.

Fig 2: Blade angle definition.

Fig. 3: Two stage fan geometry.

Fig. 4: Intake Compressor geometry.

Fig. 5: Intake - Compressor geometry grid.

Fig. 6: Lean angle definition.
Fig. 7: Leading edge meridional velocity profiles comparison between experimental data and in-viscid solver

Fig. 8: Trailing edge meridional velocity profiles comparison between experimental data and in-viscid solver

Fig. 9: Leading edge meridional velocity profiles comparison between experimental data and phenomenological module.

Fig. 10: The trailing edge meridional velocity profiles comparison between experimental data and phenomenological module

Fig. 11: Loss factor comparison - 1st stage stator

Fig. 12: Compressor characteristic - Pressure ratio for lean angle variation.

Fig. 13: The Compressor characteristic - Polytropic Efficiency for lean angle variation.

Fig. 14: Compressor characteristic - Pressure ratio for blockage factor variation.

Fig. 15: The Compressor characteristic - Polytropic Efficiency for blockage factor variation.

Fig. 16: Cumulative lean angle blockage effect on compressor performance.

\section{LIST OF TABLES}

Table 1: Two-stage fan design overall parameters [11]

Table 2: Intake Throat Boundary Conditions

\section{APPENDIX 1 - NOMENCLATURE}

\begin{tabular}{ll}
\multicolumn{2}{l}{ Abbreviations } \\
REE & Radial Equilibrium Equation \\
SLC & Streamline Curvature \\
2D & Two-Dimensional \\
& \\
Symbols & \\
\hline A, B, C & Differential equation terms \\
I & Rothalpy \\
P & Pressure \\
S & Entropy \\
T & Temperature \\
V & Absolute air velocity \\
$\mathrm{W}$ & Relative velocity \\
C & Constant of integration \\
i & Incidence Angle \\
$\mathrm{m}$ & Meridional direction \\
$\mathrm{r}$ & Radius, radial direction \\
$\mathrm{r}_{\mathrm{c}}$ & Radius of curvature \\
$\mathrm{s}$ & Tangential along the blade edge direction \\
$\mathrm{z}$ & Axial direction \\
Greek Symbols \\
\hline$\alpha$ & Absolute flow angle \\
$\beta$ & Relative flow angle \\
$\gamma$ & Sweep angle \\
$\delta$ & Deviation angle \\
$\varepsilon$ & streamline slope angle \\
\end{tabular}




$\begin{array}{ll}\lambda & \text { Lean angle } \\ \rho & \text { Density } \\ \omega & \text { Angular speed }\end{array}$

$\underline{\text { Subscripts }}$

P Pressure

$\mathrm{m} \quad$ Meridional direction

$r \quad$ Radial direction

w Whirl direction

$\mathrm{z} \quad$ Axial direction 